\title{
Manejo de Meloidogyne exigua em seringueira com produtos biológicos e químicos
}

\author{
Ewander Cristovão de Souza ${ }^{1}$, Lísias Coelho ${ }^{2} \oplus$, Ernane Miranda Lemes $^{3} \oplus$, Luciana Nunes Gontijo@ ${ }^{4}$
}

\begin{abstract}
${ }^{1}$ Engenheiro agrônomo e mestre em agronomia. Escola Técnica Estadual Professor Idio Zucchi. Rua Lúcio Sarti, 809 - Parque Eldorado, CEP 14706-120, Bebedouro - SP, Brasil. ${ }^{2}$ Professor da Universidade Federal de Uberlândia. Pós-graduação em Agronomia - PPGA. Campus Gloria UFU, Bloco:CCG, Sala:1C 206. Rod. BR 050 KM 78 CEP 38410-337, Uberlândia, MG, Brasil. ${ }^{3}$ Engenheiro agrônomo e doutor em agronomia Pós-graduação em Agronomia - PPGA. Campus Gloria UFU, Bloco:CCG, Sala:1C 206. Rod. BR 050 KM 78 CEP 38410-337, Uberlândia, MG, Brasil. ${ }^{4}$ Engenheira agrônoma e mestre em agronomia. Pós-graduação em Agronomia - PPGA. Campus Gloria UFU, Bloco:CCG, Sala:1C 206. Rod. BR 050 KM 78 CEP 38410-337, Uberlândia, MG, Brasil.
\end{abstract}

Ewander Cristovão de Souza (ewandercristovao@gmail.com)

Data de chegada: 27/06/2018. Aceito para publicação em: 08/02/2019

$10.1590 / 0100-5405 / 205581$

\section{RESUMO}

Souza, E.C; Coelho, L. Manejo de Meloidogyne exigua em seringueira com produtos biológicos e químicos. Summa Phytopathologica, v.45, n.4, p.406-412, 2019.

Com a finalidade de se conhecer melhor sobre o manejo de Meloidogyne exigua na cultura da seringueira, foram testados em campo dois métodos de controle, o químico e o biológico, da Morte Descendente da Seringueira. Assim, neste trabalho, objetivou-se avaliar a resposta de seis tratamentos: T1 - Testemunha, T2 - Paecilomyces lilacinus + Trichoderma harzianum (300 $\mathrm{g} \mathrm{ha}^{-1}$ de cada), T3 - Trichoderma asperelloides $\left(1 \mathrm{~g} \mathrm{~L}^{-1}\right)+$ Bacillus subtilis $\left(1 \mathrm{~mL} \mathrm{~L}^{-1}\right)+$ Bacillus methilotrophicus $\left(2 \mathrm{~mL} \mathrm{~L}^{-1}\right)$, T4 - Cadusafós $\left(15 \mathrm{~L} \mathrm{ha}^{-1}\right), \mathrm{T} 5-$ Bacillus subtilis + Bacillus licheniformis $\left(1,0\right.$ a $\left.1,5 \mathrm{~kg} \mathrm{ha}^{-1}\right)$ e T6 -Fluensulfone ( $\left.3 \mathrm{~L} \mathrm{ha}^{-1}\right)$, e seus potenciais de ação sobre este nematoide. Além disso, verificou-se também se houve aumento da produção de látex com os tratamentos e se há relação entre infestação e incremento diamétrico das árvores. O experimento foi conduzido em uma fazenda localizada no Triângulo Mineiro, MG. O experimento foi instalado em delineamento em blocos casualizados, com seis tratamentos e cinco repetições. Foram realizadas quatro amostragens para determinação da densidade populacional do nematoide em pré e pós aplicações, até 30 dias após a última aplicação. As amostras foram acondicionadas em sacos plásticos e levadas ao Laboratório de Nematologia da Universidade Federal de Uberlândia para o processamento. Foram avaliados o fator de reprodução, a produtividade de látex e o diâmetro médio das árvores. De todos os tratamentos utilizados, o T5 e T2 demonstraram redução do fator de reprodução em comparação aos demais. Nenhum dos produtos demonstrou eficiência quanto às três características de produtividade avaliadas: $\mathrm{kg} \mathrm{ha}^{-1}, \mathrm{~kg}$ árvore ${ }^{-1} \mathrm{e} \mathrm{kg} \mathrm{parcela}^{-1}$, indicando que ainda não se justifica a recomendação dos produtos para esta característica. Os diâmetros médios das parcelas também não foram influenciados pelos tratamentos utilizados.

Palavras-chave: Nematoide de galhas. Hevea brasiliensis. Microrganismos nematófagos. Controle biológico. Controle químico.

\section{ABSTRACT}

Souza, E.C; Coelho, L. Management of Meloidogyne exigua in rubber trees with biological and chemical products. Summa Phytopathologica, v.45, n.4, p.406-412, 2019.

To better understand the management of Meloidogyne exigua in rubber trees, two control methods for rubber tree decline, chemical and biological methods, were tested in the field. Thus, the present study evaluated the response of six treatments: T1 - Control, T2 - Paecilomyces lilacinus + Trichoderma harzianum (300 $\mathrm{g} \mathrm{ha}^{-1}$ each), $\mathrm{T} 3$ - Trichoderma asperelloides $\left(1 \mathrm{~g} \mathrm{~L}^{-1}\right)+$ Bacillus subtilis $\left(1 \mathrm{~mL} \mathrm{~L}^{-1}\right)+$ Bacillus methylotrophicus $\left(2 \mathrm{~mL} \mathrm{~L}^{-1}\right), \mathrm{T} 4$ - Cadusafos $\left(15 \mathrm{~L} \mathrm{ha}^{-1}\right)$, $\mathrm{T} 5$ - Bacillus subtilis + Bacillus licheniformis $\left(1.0\right.$ to $\left.1.5 \mathrm{~kg} \mathrm{ha}^{-1}\right)$ and T6 Fluensulfone $\left(3 \mathrm{~L} \mathrm{ha}^{-1}\right)$ and their potential action on nematodes. In addition, a possible increase in latex production due to treatments was studied, as well as a possible relationship between infestation and diameter increment in rubber trees. The experiment was conducted in a farm located in the region of "Triângulo
Mineiro", Minas Gerais State. The study was established in a randomized block design, with six treatments and five replicates. Four samplings were performed to determine nematode population density in pre and post-applications up to 30 days after the last application. The samples were stored in plastic bags and taken to the Nematology Laboratory at Federal University of Uberlândia for analysis. Reproduction factor, latex yield and mean diameter of the trees were evaluated. $\mathrm{T} 5$ and $\mathrm{T} 2$ reduced the reproduction factor, compared to the other treatments. None of the evaluated products demonstrated efficacy for the productivity characteristics: $\mathrm{kg} \mathrm{ha}^{-1}, \mathrm{~kg}$ tree $^{-1}$ and $\mathrm{kg} \mathrm{plot}^{-1}$, suggesting that recommendation of the products is not justifiable yet for this characteristic. Mean diameters of plots were not influenced by the used treatments either.

Keywords: Root knot nematode. Hevea brasiliensis. Nematophagous microorganisms. Biological control. Chemical control.

A seringueira é uma árvore de hábito ereto, podendo atingir $30 \mathrm{~m}$ de altura sob condições favoráveis, iniciando a produção de sementes aos 4 anos e a produção de látex (borracha) aos 6-7 anos quando a muda for enxertada. Esta produção pode se prolongar por 30-35 anos, com aproveitamento de madeira para processamento mecânico e energia (galhos) ao final deste período. Desenvolve-se bem em temperatura média anual de $28+2^{\circ} \mathrm{C}$, pluviosidade anual de 2.000 a $4.000 \mathrm{~mm}$, distribuída uniformemente durante todo o ano, solos com permeabilidade e profundidade adequadas e $\mathrm{pH}$ entre 4,5 e 5,5 (15).

Diante de sua grande importância econômica, quando se pensa no manejo da seringueira, deve-se dar grande atenção aos problemas fitossanitários que podem contribuir para redução na produção. Todavia, pouca atenção tem sido dada aos nematoides, mesmo já relatados os danos causados por Meloidogyne exigua Goeldi $(26 ; 28)$. Esses nematoides além de promoverem a formação de galhas e lesões, respectivamente, reduzem a absorção de água e nutrientes da planta, 
comprometendo ou, em alguns casos, até inviabilizando o cultivo das mais diversas culturas (28).

De acordo com Araújo et al. (2) o controle dos nematoides no solo pode ser feito por diferentes métodos, dando destaque aos métodos químico e biológico, que podem ser aplicados buscando sua ação como nematicidas, ou atuando na reprodução e orientação do parasita em direção às raízes da planta hospedeira no solo. Diante disso, tem crescido a aposta no controle biológico clássico que, segundo Walia (29), envolve os princípios de predação, parasitismo e patogênese. O controle biológico tem se mostrado como uma alternativa bastante viável para o manejo de fitonematoides, principalmente pela questão ambiental e por ser muitas vezes mais vantajoso economicamente, quando comparado aos métodos químicos convencionais. Existem diversos antagonistas que podem ser utilizados no manejo de nematoides e estes pertencem a vários grupos, como os fungos, bactérias, vírus e nematoides predadores (29).

Segundo Novaretti et al. (21), o controle químico é uma das importantes alternativas no país para manejo de nematoides. Já foram demonstrados resultados satisfatórios utilizando este tipo de controle (20). Apesar disto, o controle químico com nematicidas é pouco explorado em razão da sua ineficácia e dos danos ambientais causados por esses produtos (27). Objetivou-se então com esse trabalho, testar produtos biológicos e químicos para o manejo a campo da morte descendente da seringueira e a redução da densidade populacional de M. exigua; avaliar o efeito na produção de látex e determinar a relação entre infestação e incremento diamétrico das árvores.

\section{MATERIAL E MÉTODOS}

A área experimental foi selecionada em 2015 em uma fazenda localizada no Triângulo Mineiro em área de produção de látex, plantada em 2008. A distribuição das parcelas, a densidade populacional de $M$. exigua, o diâmetro das árvores e sua sobrevivência foram determinados. Este experimento foi instalado em novembro de 2016 e conduzido até julho de 2017, consistindo de 30 parcelas, em que cada uma delas continha 20 plantas ou posições de plantio, com espaçamento de $8 \mathrm{x}$ 2,5 m. O delineamento utilizado foi o de blocos casualizados, com 6 tratamentos, que estão expressos na Tabela 1, e 5 blocos, representando diferentes níveis de infestação. Todos os produtos foram aplicados dos dois lados da planta (ida e volta), utilizando um pulverizador acoplado a um trator com largura de trabalho de 6 metros com vazão de $400 \mathrm{~L} \mathrm{ha}^{-1}$. A aplicação dos produtos químicos foi em dose única e dos biológicos em três doses, com intervalos de trinta dias, exceto no T5 que foram apenas duas aplicações.

Antes da primeira aplicação foi feita uma amostragem de solo e raiz nas parcelas para estimar a população inicial de M. exigua, seguida de novas amostragens antes da segunda e terceira aplicação e, por fim, 30 dias após a terceira aplicação. Cada amostra foi composta de seis subamostras, que foram coletadas do lado direito e esquerdo de cada planta e homogeneizadas em um balde para formar a amostra composta. Após cada coleta de solo (composta de uma alíquota de aproximadamente 500 g) e raiz, a amostra foi levada ao Laboratório de Nematologia da Universidade Federal de Uberlândia para o processamento. A extração dos nematoides foi feita utilizando a metodologia de flotação centrífuga em solução de sacarose proposta por Jenkins (16). As raízes foram processadas, primeiramente, pela técnica de Hussey \& Barker (13), modificada por Boneti \& Ferraz (3), após houve o clareamento da suspensão utilizando a centrifugação pela técnica de Jenkins (16). A quantificação dos nematoides foi realizada com auxílio da câmara de Peters em microscópio fotônico.

Para a identificação da espécie de Meloidogyne foi efetuado o corte perineal e análise molecular, ambos realizados por Gontijo (12), na mesma área. A população foi a somatória de J2 no solo e ovos e/ou J2 nas raízes. A fórmula utilizada para estimar a redução populacional foi: $100-\left(\frac{P f}{P i} \times 100\right)$, onde: $P f=$ População final e $P i=$ População inicial. Para estimar a eficiência agronômica dos produtos testados em relação a testemunha, utilizou-se a fórmula de Abbott (1):

Eficiência $(\%)=\left(1-\frac{P T}{P C}\right) \times 100$, onde:

$P T$ : população tratada ou algum tratamento.

$P C$ : população na testemunha ou controle.

Os dados coletados foram submetidos a testes de pressuposições verificando a aditividade do modelo, a normalidade dos resíduos e a heterogeneidade das variâncias utilizando o programa SPSS (14). As médias dos tratamentos foram comparadas pelo teste de Scott-Knott a $5 \%$ de probabilidade. A análise estatística foi realizada utilizando-se o programa SISVAR (8).

Estimou-se a produção de látex, pesando semanalmente cada parcela, de abril a julho de 2017. Os dados de produtividade foram comparados com o programa Sisvar para fazer a correlação entre nível de controle e produtividade nas parcelas.

$\mathrm{O}$ diâmetro à altura do peito (DAP) de todas as árvores foi medido em novembro de 2015, 2016 e 2017, com suta florestal, determinandose o incremento anual. O cálculo do incremento do diâmetro das árvores foi feito subtraindo as medidas de 2017 das de 2016 [(DAP 2017) (DAP 2016)], e as de 2016 das de 2015 [(DAP 2016) - (DAP 2015)].

Tabela 1. Tratamentos, produtos, princípios ativos, doses utilizadas e modo de aplicação no experimento.

\begin{tabular}{|c|c|c|c|}
\hline Tratamento & Princípio ativo & Dose (ha) & Modo de aplicação \\
\hline 2 & $\begin{array}{l}\text { Paecilomyces lilacinus (Thom., 1910) Samson. / } \\
\text { Trichoderma harzianum Rifai, } 1969 .\end{array}$ & $300 \mathrm{~g} \mathrm{ha}^{-1}$ cada & 3 aplicações: (Nemat + Ecotrich) \\
\hline 3 & $\begin{array}{l}\text { Trichoderma asperelloides Samuels, } 2010 / \\
\text { Bacillus subtilis (Ehrenberg, 1835) Cohn, } 1872 / B . \\
\text { methylotrophicus (Madhaiyan et al., 2010). }\end{array}$ & $\begin{array}{l}1 \mathrm{~g} \mathrm{~L}^{-1} \\
2 \mathrm{ml} \mathrm{L}^{-1} \\
1 \mathrm{ml} \mathrm{L}^{-1} \\
\end{array}$ & $\begin{array}{l}3 \text { aplicações: } \\
\text { (GF } 422+\text { Onix / GF } 422+\text { Rizos / GF } 422 \\
+ \text { Onix) }\end{array}$ \\
\hline 4 & Cadusafós & $15 \mathrm{~L} \mathrm{ha}^{-1}$ & Dose única (Rugby) \\
\hline 5 & $\begin{array}{l}\text { Bacillus subtilis / } \\
\text { B. licheniformis (Weigmann, 1898) Chester, } 1901 .\end{array}$ & $1-1,5 \mathrm{Kg} \mathrm{ha}^{-1}$ & 3 aplicações (Nemix) \\
\hline 6 & Fluensulfone & $3 \mathrm{~L} \mathrm{ha}^{-1}$ & Dose única (Nimitz) \\
\hline
\end{tabular}




\section{RESULTADOS E DISCUSSÃO}

Os dados de população inicial em 2015 foram agrupados para divisão dos tratamentos, criando um ambiente homogêneo de distribuição, uma vez que em campo as condições são heterogêneas. Em novembro de 2016 e abril de 2017 não houve diferença entre as populações de $M$. exigua nos tratamentos aplicados (Tabela 2). O fator de reprodução (FR) de $M$. exigua foi menor em T5 (Nemix) e T6 (Nimitz).

Houve diferença populacional entre as duas épocas avaliadas e isso pode ter sido atribuído à redução da precipitação, visto que os níveis populacionais diminuíram tanto nos tratamentos, como na testemunha. As médias históricas de temperatura e precipitação no município onde o experimento foi instalado corroboram esta hipótese (Figura 1). Isso porque o patógeno tem seu desenvolvimento reduzido com a falta de umidade.

Não houveram diferenças nos tratamentos dentro de cada época quanto à densidade populacional. Quando avaliada a densidade populacional inicial (DPI), já era esperado, pois a divisão das parcelas no experimento foi exatamente para criar esse ambiente homogêneo. Porém, ao se avaliar a densidade populacional final (DPF), nota-se que nenhum dos produtos foi superior na redução populacional do nematoide. O manejo de nematoides em culturas anuais deve ser pensado para a próxima safra, não sendo indicado se a cultura estiver no campo. No caso de culturas perenes, que é o caso da seringueira, intervenções devem ser feitas, uma vez que os danos se tornam cada vez mais acentuados graças à rápida reprodução do nematoide (22). Fazer intervenções com a cultura instalada, neste caso, é algo que dificulta

Tabela 2. Densidade populacional de Meloidogyne exigua antes e ao final do ensaio e Fator de Reprodução. Uberlândia, MG. 2018.

\begin{tabular}{lccc}
\hline Tratamentos $^{1}$ & ${ }^{2}$ DPI - novembro 2016 & ${ }^{3}$ DPF - abril 2017 & Fator de Reprodução \\
\hline 1. Testemunha & $33.301,5 \mathrm{a}$ & $11.670,5 \mathrm{a}$ & $0,46 \mathrm{~b}$ \\
2. Nemat + Ecotrich & $20.395,9 \mathrm{a}$ & $9.176,5 \mathrm{a}$ & $0,45 \mathrm{~b}$ \\
3. Onix / Rizos / GF 422 & $22.454,0 \mathrm{a}$ & $10.465,3 \mathrm{a}$ & $0,45 \mathrm{~b}$ \\
4. Rugby & $23.620,7 \mathrm{a}$ & $9.018,2 \mathrm{a}$ & $0,42 \mathrm{~b}$ \\
5. Nemix & $25.293,8 \mathrm{a}$ & $6.146,6 \mathrm{a}$ & $0,24 \mathrm{a}$ \\
6. Nimitz & $26.828,9 \mathrm{a}$ & $4.892,9 \mathrm{a}$ & $0,19 \mathrm{a}$ \\
\hline Média & $25.315,8 \mathrm{~B}$ & $8.561,7 \mathrm{~A}$ & \\
\hline CV (\%) & 37.86 & 34.86 & 43,32 \\
F & 4,759 & 0,732 & 1,799 \\
$\mathrm{~F}_{\text {tukey }}$ & 5,190 & 0,132 & 4,852 \\
W & 0,961 & 0,989 & 0,953
\end{tabular}

${ }^{1}$ Médias seguidas pela mesma letra maiúscula nas linhas e minúsculas nas colunas, não diferem entre si pelo teste de Scott-Knott a 0,05 de significância. CV: coeficiente de variação; F: estatística do teste de Levene; $\mathrm{F}_{\text {tukey }}$ : estatística do teste de aditividade; W: estatística do teste de Shapiro-Wilk. ${ }^{2} \mathrm{DPI}=\mathrm{Densidade}$ populacional inicial -número de nematoides por grama de raiz e por $150 \mathrm{~cm}^{3}$ de solo. ${ }^{3} \mathrm{DPF}=$ Densidade populacional final -número de nematoides por grama de raiz e por $150 \mathrm{~cm}^{3}$ de solo.

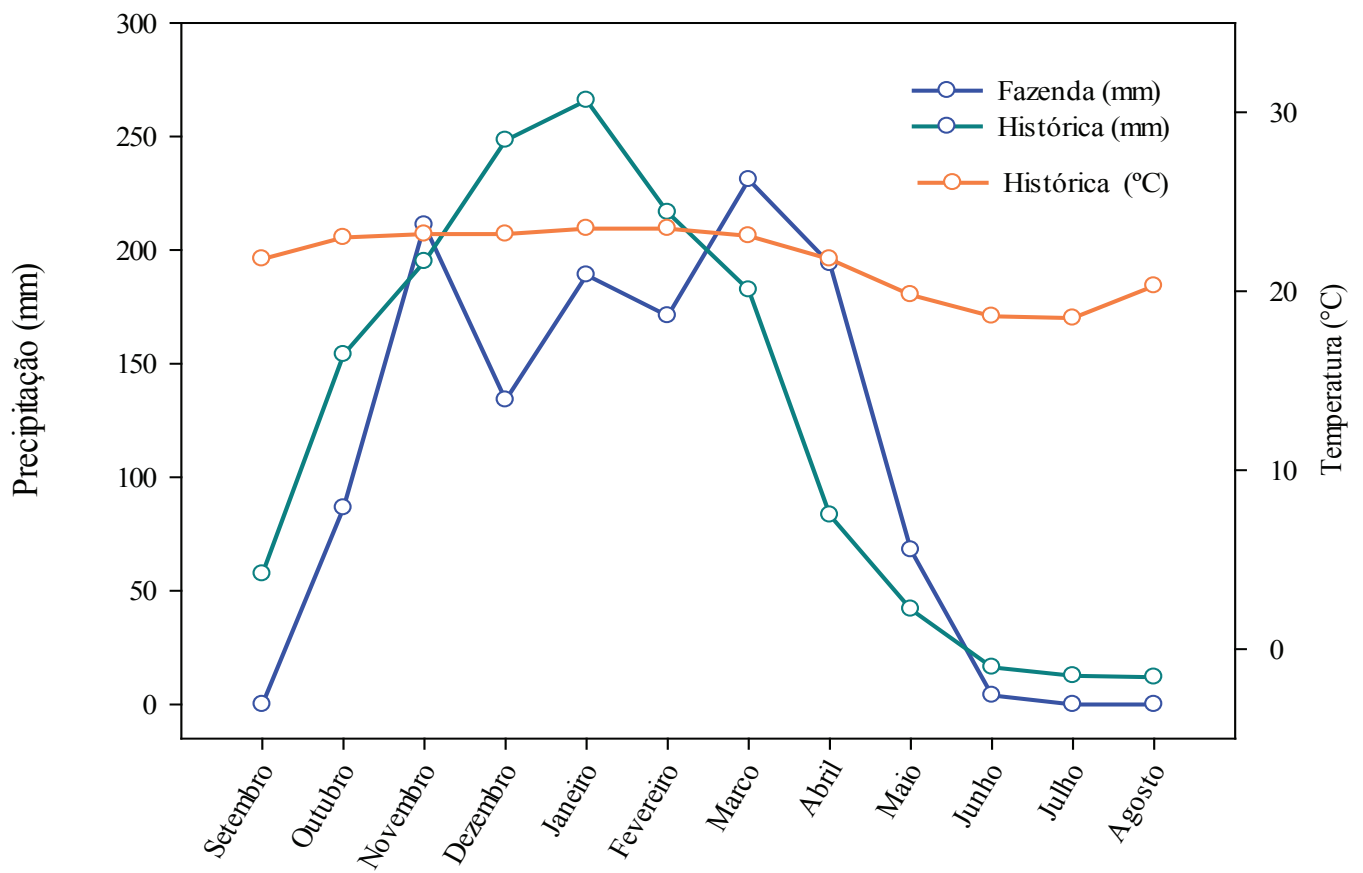

Figura 1. Dados históricos de temperatura e precipitação no município onde a área experimental está instalada. Triângulo Mineiro. Fonte: Alvares et al. (2014). 
o controle, pois $M$. exigua se aloja dentro da raiz da planta, sem expor sua parte posterior e sua massa de ovos (25), contribuindo para que ele esteja protegido contra a ação dos agentes nematicidas.

Analisando a Tabela 3, constata-se que os tratamentos T5 (Nemix) e T6 (Nimitz) apresentaram comportamento semelhante no que se refere à redução populacional. T6 (Nimitz) obteve a melhor eficiência relativa de controle, seguido de T5 (Nemix). Quanto à eficiência de Abbott, os resultados são semelhantes, apesar de nenhum dos produtos poderem ser nomeados como eficazes, uma vez que (8) classifica como eficazes os produtos que apresentam pelo menos $80 \%$ de controle.

Tabela 3. Porcentagem de redução relativa (\%) dos tratamentos, tendo como base a testemunha (eficiência relativa) e eficiência de Abbott após o controle químico e biológico. Uberlândia, MG. 2018.

\begin{tabular}{lcc}
\hline Tratamentos & Eficiência relativa (\%) & Eficiência Abbott (\%) \\
\hline Testemunha & 100 & - \\
Nemat + Ecotrich & 102 & 21,37 \\
Onyx / Rizos / GF 422 & 102 & 10,33 \\
Rugby & 109 & 22,72 \\
Nemix & 148 & 47,34 \\
Nimitz & 159 & 58,08 \\
\hline
\end{tabular}

Carvalho et al. (5) já haviam demonstrado a eficiência superior de Fluensulfone na redução da reprodução do nematoide, quando comparado com Cadusafós. Diferentes doses e tecnologias de aplicação de Fluensulfone foram testadas por Corte et al. (7) em condições de campo em café, e os autores demonstraram que Cadusafós e Fluensulfone se comportaram de maneira semelhante e efetiva na redução da reprodução do nematoide, o que difere do presente trabalho. Os autores demonstraram ainda, que Fluensulfone, quando aplicado em faixa de $50 \mathrm{~cm}$, em ambos os lados da planta e com a limpeza prévia da saia do café, foi o tratamento que obteve melhores resultados. Esta aplicação foi semelhante à utilizada neste estudo, o que demonstra a importância de se seguir o protocolo de aplicação para otimização dos resultados. Fluensulfone é um princípio ativo bastante promissor, pois possui um mecanismo de ação diferente dos carbamatos, sendo conhecido como um verdadeiro nematicida, pois atua de forma seletiva, matando apenas nematoides, afetando sua reprodução, desenvolvimento, alimentação e motilidade (17).

Carvalho (4) testou os efeitos de Cadusafós em tomateiro e em condições de casa de vegetação sobre $M$. javanica e $M$. incognita. O mesmo foi utilizado como controle positivo, demonstrando alta eficiência na redução do Fator de Reprodução, sendo recomendando em ensaios posteriores. Marcuzzo et al. (19), embora não tenham observado diferenças significativas entre seus tratamentos para controle de $M$. incognita e $M$. exigua com diferentes doses de Cadusafós, observaram, pelo fator de reprodução, que algumas doses do produto proporcionaram os menores níveis populacionais aos 270 dias após sua aplicação. Eles atribuíram a não eficácia dos tratamentos à alta população inicial das duas espécies de nematoides presentes na área e à elevada agressividade e multiplicação de $M$. incognita em cafeeiro. Isso demonstra que os resultados com Cadusafós ora são promissores, ora não. Neste trabalho pode-se atribuir a ineficiência deste produto a alguns fatores, como: as condições climáticas e ambientais (chuva e serapilheira), que podem ter interferido na ação do produto ou evitado que o mesmo tivesse contato com os nematoides, visto que Cadusafós é um nematicida de contato e ingestão (11) e estes eventos não ocorrem em experimentos em casa de vegetação. Outro fator que deve ser levado em consideração é a aplicação. A recomendação de aplicação do produto em café, que também é uma cultura perene, é aplicar em sistema meia lua, dos dois lados da planta na projeção da copa e cobrir com terra com equipamento adequado (11). No presente trabalho, o nematicida não foi coberto com terra, por isso podem ter havido perdas. Recomenda-se que o produto seja aplicado no solo na capacidade de campo (11). O mesmo foi aplicado em época chuvosa, em que o solo pode ter excedido a capacidade de campo e havido mais perdas do produto.

De modo geral, Bacillus subtilis e B. methylotrophicus são nematicidas microbiológicos eficazes no controle de fitonematoides no solo, em qualquer cultura no qual ocorram (4). Diante disso, chega-se à conclusão que os produtos não apresentaram efeitos na redução do Fator de Reprodução, pois eles apresentam eficiência em nematoides quando os mesmos estão no solo, e no caso do presente estudo, grande parte dos nematoides estava dentro das raízes das plantas, onde os produtos não alcançam, devido à proteção que estas proporcionam a estes patógenos (25). Pelo fato de a seringueira ser uma cultura perene, não é possível fazer com frequência a reforma da área e tratamento do solo com nematicidas. É importante ressaltar que o período analisado foi de novembro de 2016 a abril de 2017 (período chuvoso) e, por isso, a seringueira ainda não havia perdido raízes pela falta de chuva e os nematoides não tinham migrado para solo em busca de novas raízes. Ferreira (10) testou três gêneros de Bacillus para controle biológico de $M$. incognita e $M$. javanica in vitro e em vasos a céu aberto com mudas de cana de açúcar e Cadusafós como controle químico. De modo geral, o autor constatou controle dos nematoides in vitro pela redução da eclosão de $\mathrm{J} 2$ de $M$. javanica em um dos tratamentos, que se comportou como o tratamento químico. Já na motilidade dos J2, os demais causam a mortalidade, mas foram inferiores ao tratamento químico. Em M. incognita, Cadusafós foi o único que obteve bons resultados. No ensaio em vasos, nenhum dos tratamentos com Bacillus obteve controle dos nematoides, similarmente aos resultados obtidos em T3 (Onix + Rizos + GF422) deste trabalho.

Estes resultados também são semelhantes aos encontrados em T5 (Nemix), pois os produtos que são à base de Trichoderma e Bacillus, não apresentaram potencial na diminuição do Fator de Reprodução, como também constatado por Carvalho (4), em um dos tratamentos, contendo a mistura de dois produtos que tinham como ingredientes ativos um gênero de Bacillus e um de Trichoderma, no controle dos nematoides $M$. javanica e $M$. incognita no tomateiro. Este tratamento, segundo a autora, não foi promissor, tanto pela sua época de aplicação como pela quantidade de produto aplicado, o que mostra que a associação dos dois microrganismos nem sempre é eficaz. Chaves (6) encontrou que, em geral, a presença de Trichoderma não resultou em mudas de melhor qualidade, quando comparados à testemunha, porém, houve redução no número de galhas e massas de ovos de nematoides nos tratamentos inoculados com os isolados do fungo. A mesma autora comprovou o potencial antagônico de Trichoderma no controle de Meloidogyne enterolobii em mudas de alface.

Em T2 (Nemat + Echotrich) os resultados diferiram dos encontrados por Santiago et al. (24) que, ao avaliarem a patogenicidade de $P$. lilacinus sobre $M$. paranaensis em cafeeiro na condição de casa de vegetação, encontraram resultados satisfatórios em três dos dez isolados 
testados. Esta diferença ocorreu, provavelmente, porque os autores, ao iserirem as mudas de cafeeiro nos vasos infestados com inóculo do nematoide, já haviam feito uma pré-inoculação dos isolados de $P$. lilacinus, o que fez com que os mesmos tivessem ação no nematoide antes de sua penetração nas raízes da planta, ou seja, antes dele estar protegido dentro da mesma. Vale ressalar que houve similaridade aos resultados de Robl et al. (23), que demonstraram em seu estudo com isolados de $P$. lilacinus a ineficiência no controle reprodutivo de $M$. incognita raça 3 e $M$. javanica na cultura do tomate.

De modo geral, a ineficiência de alguns produtos diante dos tratamentos pode ser atribuída ao fato de que durante as aplicações foi constatada a presença de serapilheira e plantas daninhas, que podem ter servido de barreira para que os produtos atingissem seu alvo, uma vez que a efetividade de nematicidas, sejam biológicos ou químicos, depende exclusivamente de atingirem seu alvo biológico. Além disto,
M. exigua fica completamente abrigado dentro da raiz (25), oferecendo menos oportunidades para o parasitismo pelos agentes de controle biológico, ou pelos produtos químicos.

Com os dados das coletas de novembro de 2016 e janeiro, março e abril de 2017, foi possível avaliar o progresso da população de nematoides no tempo. Os gráficos ilustram as alterações que ocorreram após as aplicações em cada mês. Para fins didáticos, foram divididos os tratamentos em um gráfico com produtos biológicos e outro com os químicos.

Apesar de em geral os produtos biológicos terem se mostrado ineficientes nos índices populacionais, ao se observar o gráfico de tendências, pode-se concluir que T5 (Nemix) se mostra promissor na diminuição dos mesmos em relação aos outros produtos biológicos testados, que tiveram comportamento similar aos da testemunha (Figura 2). O T5 (Nemix), de novembro a janeiro, não demonstrou influência

Tabela 4. Produção de látex em seringueira submetida a nematicidas químicos e biológicos.

\begin{tabular}{lccc}
\hline Tratamento $^{1}$ & Produção por parcela (kg) & Produção por árvore (kg) $\left.^{\text {Produtividade (kg ha }}{ }^{-1}\right)$ & $1.267,86 \mathrm{a}$ \\
\hline Testemunha & $50,71 \mathrm{a}$ & $4,73 \mathrm{a}$ & $1.181,50 \mathrm{a}$ \\
Nemat + Ecotrich & $47,26 \mathrm{a}$ & $4,48 \mathrm{a}$ & $1.290,84 \mathrm{a}$ \\
Onix / Rizos / GF 422 & $51,63 \mathrm{a}$ & $5,21 \mathrm{a}$ & $1.199,33 \mathrm{a}$ \\
Rugby & $47,97 \mathrm{a}$ & $4,54 \mathrm{a}$ & $1.333,75 \mathrm{a}$ \\
Nemix & $53,35 \mathrm{a}$ & $4,97 \mathrm{a}$ & $1.202,38 \mathrm{a}$ \\
Nimitz & $48,09 \mathrm{a}$ & $4,42 \mathrm{a}$ & 18,01 \\
\hline CV (\%) & 18,01 & 11,9 & 1,645 \\
F & 0,548 & 0,548 & 3,520 \\
$\mathrm{~F}_{\text {tukey }}$ & 2,010 & 2,010 & 0,932 \\
W & 0,903 & 0,903 & \\
\hline
\end{tabular}

${ }^{1}$ Médias seguidas por letras distintas na coluna, diferem entre si pelo teste de Scott-Knott a 0.05 de significância. CV: coeficiente de variação; F: estatística do teste de Levene; $\mathrm{F}_{\text {tukey }}$ : estatística do teste de aditividade; W: estatística do teste de Shapiro-Wilk.

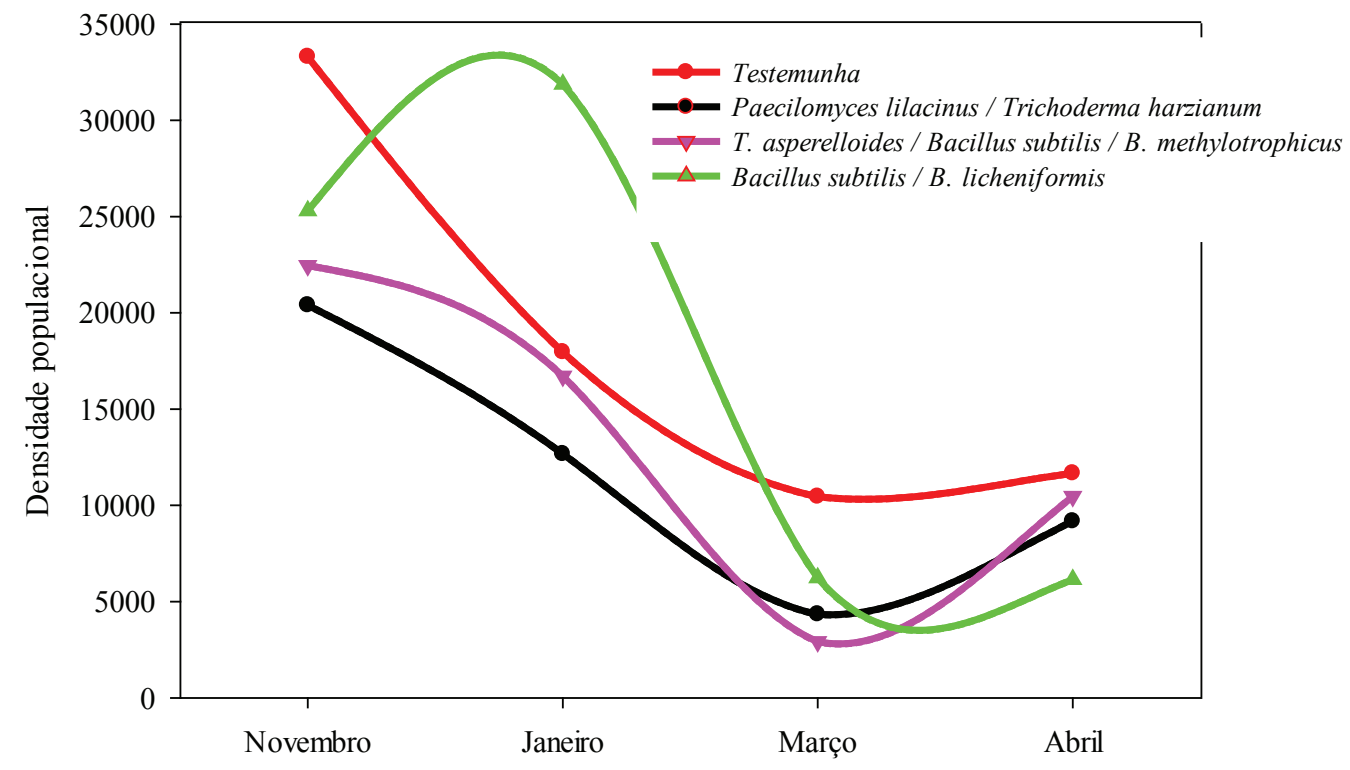

Figura 2. Variação populacional de Meloidogyne exigua entre novembro de 2016 e abril de 2017 após tratamento com produtos biológicos. Uberlândia, MG. 2018. 


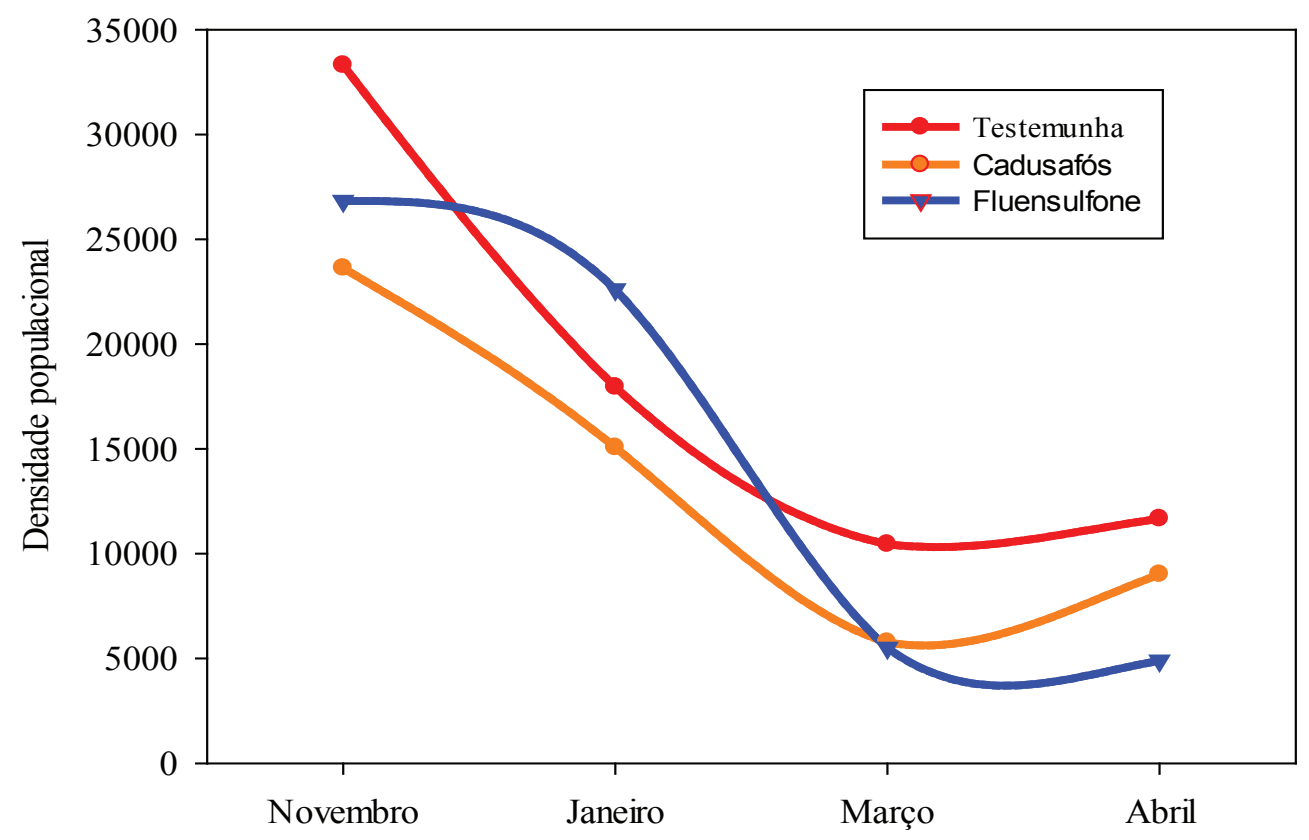

Figura 3. Variação populacional de Meloidogyne exigua entre novembro de 2016 e abril de 2017 após tratamento com produtos químicos. Uberlândia, MG. 2018.

Tabela 5. Médias de incrementos dos diâmetros por tratamento (cm).

\begin{tabular}{|c|c|c|}
\hline Tratamento $^{1}$ & Incremento 2016 & Incremento 2017 \\
\hline Testemunha & $0.65 \mathrm{a}$ & $1.07 \mathrm{a}$ \\
\hline Nemat + Ecotrich & $0.62 \mathrm{a}$ & $0.80 \mathrm{a}$ \\
\hline Onyx / Rizos / GF 422 & $0.55 \mathrm{a}$ & $1.05 \mathrm{a}$ \\
\hline Rugby & $1.20 \mathrm{a}$ & $0.90 \mathrm{a}$ \\
\hline Nemix & $1.10 \mathrm{a}$ & $1.32 \mathrm{a}$ \\
\hline Nimitz & $1.92 \mathrm{a}$ & $0.97 \mathrm{a}$ \\
\hline Média & $1.00 \mathrm{~A}$ & $1,01 \mathrm{~A}$ \\
\hline CV (\%) & 33,18 & 136,70 \\
\hline $\mathbf{F}$ & 2,477 & 2,022 \\
\hline $\mathbf{F}_{\text {tukey }}$ & 7,433 & 0,784 \\
\hline $\mathbf{W}$ & 0,890 & 0,946 \\
\hline
\end{tabular}

${ }^{1}$ Médias seguidas pela mesma letra maiúscula nas linhas e minúsculas nas colunas, não diferem entre si pelo teste de Scott-Knott a 0.05 de significância. CV: coeficiente de variação; F: estatística do teste de Levene; $\mathrm{F}_{\text {tukey }}$ : estatística do teste de aditividade; W: estatística do teste de Shapiro-Wilk.

na população de nematoides, uma vez que seus índices aumentaram. Após janeiro, há uma queda brusca de população de nematoide que se estende até a última amostragem realizada em abril. A demora na ação do produto pode ser atribuída ao fato de que um produto biológico tem seu efeito mais lento.

Os produtos químicos em geral também mostraram pouco efeito na redução populacional do nematoide (Figura 3). Apesar disso, quando se avalia o gráfico de tendências, conclui-se que em T6 (Nimitz) houve um comportamento mais desejado, pois foi reduzindo gradativamente a população de nematoides, que também ocorreu aos outros tratamentos, entretanto, de forma diferente. Essa redução deu-se até março e se estabilizou até abril (último mês avaliado), mostrando potencial de redução permanente no patógeno.

Quanto à produtividade, nota-se que, nesta safra de colheita de látex (2016-2017), entre abril e julho, não houve incrementos em relação aos tratamentos que justificassem a recomendação dos mesmos a campo (Tabela 4). Espera-se obter respostas nas próximas safras, ao passo que também se espera maior eficiência de controle da população de M. exigua com os produtos.

Apesar de não ter havido diferenças estatísticas entre os tratamentos, o potencial produtivo está seriamente comprometido, já que Machado (18) demonstrou que outra área na mesma propriedade obteve produtividade de $1.622,00 \mathrm{~kg} \mathrm{ha}^{-1}$. Esta produtividade é bem superior à observada neste experimento, reforçando a imediata necessidade de serem adotadas medidas de manejo deste importante fitopatógeno.

Novaretti et al. (20) encontraram aumento na produção de grãos em soja quando testaram o controle químico de nematoides, diferentemente dos resultados encontrados no presente estudo, provavelmente pela diferente fisiologia das plantas, que influencia na resposta aos tratamentos e por seus produtos (café o fruto, soja o grão, cana o colmo e seringueira o látex) serem completamente diferentes e também pelo fato de que os autores fizeram a aplicação dos produtos no plantio, permitindo que o mesmo agisse no nematoide no solo, antes deste penetrar na raiz. Já Novaretti et al. (21), não encontraram acréscimos em produtividade de cana quando testaram produtos químicos no controle de $M$. incognita e P. zeae, de maneira semelhante ao encontrado no presente estudo.

Analisando os dados da Tabela 5, nota-se que, independente do tratamento adotado, não foram constatadas diferenças significativas nas médias dos incrementos diamétricos das árvores. Isso demonstra que o crescimento das árvores não foi influenciado pelos produtos e nem pelo fator tempo.

Pode-se concluir que, após os tratamentos, os produtos Nemat + Ecotrich, Onix + Rizos + GF 422, Rugby, Nemix e Nimitz, não demonstraram potencial de redução da população final. Conclui-se também que fatores ambientais, como a precipitação, são grandes influenciadores de redução natural da população de nematoides.

Quanto ao fator de reprodução, nos tratamentos com Nimitz e Nemix, observou-se uma redução no mesmo e uma eficiência de controle superior aos demais. 
Nas características relacionadas à produtividade, não houveram diferenças que justificassem a recomendação de controle para tal característica.

Quando avaliado o incremento diamétrico, depreende-se que também não foram encontradas respostas que correlacionem o aumento da característica em relação aos tratamentos.

Diante dos resultados, recomendam-se mais estudos com controle de nematoides em seringueira, visando obter respostas positivas a longo prazo.

\section{AGRADECIMENTOS:}

Às empresas FMC, Ballagro, Grupo Farroupilha e ADAMA por disponibilizarem os produtos para a realização do trabalho.

\section{REFERÊNCIAS}

1. ABBOTT, W. S. A method of computing the effectiveness of an insecticide. Journal of Economic Entomology, Lanham, v. 18, n. 2, p. 265-267, 1925.

2. ARAÚJO, F. F.; SILVA, J. F. V; ARAÚJO, A. S. F. Influência de Bacillus subtilis na eclosão, orientação e infecção de Heterodera glycines em soja. Ciência Rural, Santa Maria, v. 32, n. 2, p.197-202, jan. 2002.

3. BONETI, J. I. S.; FERRAZ, S. Modificação do método de Hussey e Barker para extração de ovos de Meloidogyne exigua do cafeeiro. Fitopatologia Brasileira, Brasília, v.6, n.3, p.553, 1981.

4. CARVALHO, P. H. Controle biológico e alternativo de Meloidogyne incognita e M. javanica em tomateiro. 2017. 98 f. Dissertação (Mestrado) - Curso de Programa de Pós-graduação em Fitopatologia, Universidade de Brasília, Brasília, 2017. Disponível em: $<$ http://repositorio.unb.br/bitstream/10482/23402/1/2017_ PatríciaHonoratodeCarvalho.pdf $>$. Acesso em: 01 fev. 2018.

5. CARVALHO, B. G.; FERNANDES, A. L. T.; MOSCA, E.; LIMA, K. C. C.; FERREIRA, R.T. Eficácia do fluensulfone aplicado via gotejo no controle de nematoides da cultura do café. Araguari: FeniCafé. 2018. Disponível em: $<$ http://fenicafe.com.br/assets/uploads/aprovados/5.pdf $>$. Acesso em: 10 jan. 2018

6. CHAVES, P. P. N. Qualidade de mudas de alface inoculadas com Trichoderma e reação de plantas adultas de alface a nematoides de galhas na presença de Trichoderma. 2015. 144 f. Dissertação (Mestrado em Produção Vegetal). Universidade Federal do Tocantins, Gurupi, 2015.

7.CORTE, G. D.; FARIA, D. S.; BENETTI, E.; BRITES, M.; VIEIRA, H. Eficiência do novo nematicida fluensulfone 480)no controle de Meloidogyne exigua em Coffee arabica $\mathbf{L}$. In: Congresso Brasileiro de Pesquisas Cafeeiras, 40, 2014, Serra Negra. Anais... Brasília: Embrapa Café, 2014. 2 p. Disponível em: <http://www.sbicafe.ufv.br/bitstream/handle/123456789/6486/279_40CBPC-2014.pdf?sequence=1>. Acesso em: 15 fev. 2018.

8. EMBRAPA. Normas para avaliação e para a indicação de inseticidas. 2018. Disponível em: $<$ https://www.cpao.embrapa.br/aplicacoes/cd_trigo/Regimento/ normas\%20inseticidas.pdf $>$. Acesso em: 10 jan. 2018.

9. FERREIRA, D. F. Sisvar: a computer statistical analysis system. Ciência e Agrotecnologia, Lavras, v. 35, n.6, p. 1039-1042, 2011.

10.FERREIRA, R. J. Espécies de Bacillus no controle de Meloidogyne incognita e Meloidogyne javanica in vitro e na cana de-açúcar. 2015. 72 f. Dissertação (Mestrado em Agronomia - Produção Vegetal), Faculdade de Ciências Agrárias e Veterinárias - Unesp, Jaboticabal, 2015. Disponível em: <https://repositorio. unesp.br/bitstream/handle/11449/124080/000829026.pdf?sequence=1>. Acesso em: 04 set. 2017

11. FMC (Brasil). Relatório do Produto (Rugby). S.l. 2017. Disponível em: $<$ https://www.fmcagricola.com.br/bula_geraPDF.aspx?cod=45>. Acesso em: 18 jan. 2018.

12. GONTIJO, L. N. Meloidogyne exigua: distribuição espacial, nível de infestação, mortalidade de seringueiras e controle com plantas antagonistas. 2017. 49 f. Dissertação (Mestrado em Agronomia) - Universidade Federal de Uberlândia, Uberlândia, 2017. Disponível em: <https://repositorio.ufu.br/ bitstream/123456789/19442/1/MeloidogyneExiguaDistribuicao.pdf $>$. Acesso em: 20 jan. 2018.
13. HUSSEY, R. S.; BARKER, K. R. A comparison of methods for collecting inocula of Meloidogyne spp. including a new technique. Plant Disease Reporter, Washington, DC. v.57, n.12, p.1025-1028, Dec. 1973.

14. IBM Corporation. IBM SPSS Statistics 20. IBM Corporation, Armonk, NY: IBM corp. 2011

15. MARTO, G.B.T. Hevea brasiliensis (Seringueira). 2007. Piracicaba: Instituto de Pesquisas e Estudos Florestais. Disponível em: $<$ http://www.ipef.br/identificacao/hevea.brasiliensis.asp>. Acesso em: 19 out. 2017.

16. JENKINS, W. R. A rapid centrifugal - flotation technique for separating nema^ todes from soil. Plant Disease Reporter, Washington, DC, v. 48, p. 692, 1964.

17. KEARN, J.; LUDLOW, E.; DILLON, J.; O'CONNOR, V.; HOLDEN-DYE, L. Fluensulfone is a nematicide with a mode of action distinct from anticholinesterases and macrocyclic lactones. Pesticide Biochemistry and Physiology, San Diego, v. 109, p. 44-57, 2014.

18. MACHADO, T. V. Alterações de componentes silviculturais e da produção de Hevea brasiliensis causados por Meloidogyne exigua. 2018. $43 \mathrm{f}$. Dissertação (Mestrado em Agronomia). Universidade Federal de Uberlândia, Uberlândia. 2018.

19. MARCUZZO, K. V; SANTOS, M. A; JULIATTI, F. C; MELO, B; SEVERINO, G. M. In: SIMPÓSIO DE PESQUISA DOS CAFÉS DO BRASIL, 1., 2000, Poços de Caldas. Anais... Brasília: Embrapa Café, 2000. v. 2, p. 360 - 365. Disponível em: <http://www.sbicafe.ufv.br/bitstream/handle/123456789/688/155537 Art070f.pdf?sequence=1\&isAllowed $=y>$. Acesso em: 07 out. 2017.

20. NOVARETTI, W. R. T.; MIRANDA, M. A. C.; ALCÂNTARA, V.S.B. Tratamento químico visando o controle de nematoides em soja. Nematologia Brasileira, Piracicaba, v. 5, n. 2, p.247-255, 1981.

21. NOVARETTI, W. R. T.; MONTEIRO, A. R.; FERRAZ, L. C. C. B. Controle químico de Meloidogyne incognita e Pratylenchus zeae em cana de açúcar com carbofuran e terbufos. Nematologia Brasileira, Piracicaba, v. 22, n. 1, p.60-74, dez. 1998.

22. O PREJUÍZO dos nematoides é assustador. Produção de Jaime Maia dos Santos. [S.1]: FMC. 2017. 1 video (4.49 min.). Disponível em: $<$ https://www.youtube. com/watch? $v=h 9$ CFEbtnlUY\&t=11s $>$. Acesso em: 12 jan. 2017.

23. ROBL, D.; MACEDA, A; DALZOTO, P. R.; SENKIV, C.C.; PIMENTEL, I. C.; ZAWADNEAK, M. A. C. Controle de nematoides das galhas em plantas de tomate com isolados mutantes de Paecilomyces lilacinus. Iniciação Científica Cesumar, Maringá, v. 14, n. 2, p.213-219, jul/dez. 2012. Disponível em: $<$ http:// periodicos.unicesumar.edu.br/index.php/iccesumar/article/view/1897>. Acesso em: 28 ago. 2017

24. SANTIAGO, D. C.; CADIOLI, M. C.; OLIVEIRA, A. D.; PAES, V. S.; ARIEIRA, G. O. Avaliação da patogenicidade de Paecilomyces lilacinus sobre Meloidogyne paranaensis em cafeeiro. In SIMPÓSIO DE PESQUISA DOS CAFÉS DO BRASIL, 5.2007. Águas de Lindóia. Anais... Brasília: Embrapa Café. p. 1-5. 2007. Disponível em: http://www.sapc.embrapa.br/arquivos/consorcio/ spcb_anais/simposio5/p273.pdf. Acesso em: 08 dez. 2017.

25. SANTOS, J. M. Estudos das principais espécies de Meloidogyne Goeldi que infectam o cafeeiro no Brasil com descrição de Meloidogyne goeldii sp.n. 1997. 153 f. Tese (Doutorado em agronomia). UNESP-FCA, Botucatu, 1997.

26. SANTOS, J. M.; MATTOS, C.; BARRÉ, L.; FERRAZ, S. Meloidogyne exigua, sério patógeno da seringueira nas plantações E. Michelin, em Rondonópolis, MT. In: CONGRESSO BRASILEIRO DE NEMATOLOGIA, 16., 1992, Lavras. Anais... Lavras: Sociedade Brasileira de Fitopatologia, 1992. v. 17, p. 75.

27. SUASSUNA, N; FERREIRA, A; MORELLO, C. Eficiência de nematicida e resistência genética no controle do nematoide das galhas. In: CONGRESSO BRASILEIRO DE ALGODÃO, 9., 2013, Brasília. Trabalhos apresentados. Brasília: Ampa, 2013. Disponível em: <https://ainfo.cnptia.embrapa.br/digital/ bitstream/item/104534/1/fito-nema120017.pdf $>$. Acesso em: 18 out. 2017.

28. TAKAHASHI, V. S. P. Inter-relações entre nematoides, fungo e a cultura da seringueira. 2015.98 f. Tese (Doutorado em Produção Vegetal) - Universidade Estadual Paulista, Jaboticabal, 2015. Disponível em: $<$ https://repositorio.unesp. $\mathrm{br} / \mathrm{bitstream} /$ handle/11449/128165/000849022.pdf?sequence=1>. Acesso em: 10 ago. 2017.

29. WALIA, R. K. Biological Control - Recapitulating the basic principles. Indian Journal of Nematology, New Delhi, v. 39, n 2, p. 129-137. dez. 2009. Disponível em: $<$ http://nematology.in/data/documents/V39N02P129.pdf $>$. Acesso em: 03 out. 2017. 